\title{
Assessment of structural bearing capacity in case of internal explosion
}

\author{
Vyacheslav Gorev and Evgeniya Chelekova* \\ Moscow State University of Civil Engineering, Yaroslavskoe shosse, 26, Moscow, 129337, Russia
}

\begin{abstract}
The work has developed a method for determining the deformation of bent rod structures during an internal explosion. When consideration of a quasi-stationary explosion, a model load is proposed, taking into account the pressure rise section to the maximum value at the beginning of the explosion, then a stationary section and then a decline. The pressure in the stationary area is equal to more of the two peaks. With a sufficiently long stationary section, the maximum deformation is realized here, and is also determined from the energy balance. In the case of a short section of stationary pressure, maximum deformation develops towards the end of the explosion after a pressure drop. The solutions are suitable for describing deformation of beams with different fastening at the ends and are limited to the case when the maximum value of the load does not exceed the resistance of the structure. The results of the work can be used in assessing the load capacity of elements of explosive industries, residential premises, taking into account the action of protective structures.
\end{abstract}

\section{Introduction}

Internal emergency explosions continue to occur with regular frequency. The devastating effects of these explosions indicate the poor effectiveness of existing measures to protect construction facilities [1-5]. When analyzing these measures, internal explosions should be distinguished by their nature and the mechanism of their course. Most of these explosions are quasi-static, when it can be assumed that the pressure in the room, at every moment of time, is the same at all its points of volume, but changes only in time. Such explosions are typical for industrial enterprises with hydrocarbon handling and for residential premises. In case of external emergency explosions, the calculation of buildings for bearing capacity is carried out using model loads [6-8]. With internal quasi-static, there is no model load. This is due to the influence of safety structures, the action of which greatly complicates the issue. To date, this feature has not been taken into account. In [9-10], the actual dynamic load is replaced by the equivalent static load. The value of the dynamic factor is not

\footnotetext{
* Corresponding author: SalymovaEY@mgsu.ru
} 
justified. In [8] model load shall be built on the basis of rough ideas about pressure dynamics during explosion in closed and depressurized volume. The impact of opening openings using safety structures is not taken into account. In particular, it is accepted that the increase in pressure in a closed volume during a quasi-static explosion follows the law of $\Delta \mathrm{P} \sim \mathrm{t}^{5}$, up to the end of the explosion, that the time of the explosion in a closed and depressurized volume is the same, that the expiration of hot products of the explosion does not affect its character. All these assumptions contradict known experimental factors $[11,14]$. The dependencies used to determine the parameters of the explosion are not explained, and are not accompanied by references. Thus, at present there are no model explosive loads on which the effects of an internal explosion can be calculated, as in the case of an external explosion [6-8].

As a result, there is an urgent need to develop model explosive loads during an internal explosion, which adequately take into account the characteristics of the internal explosion, both taking into account the influence of opening openings, and taking into account the influence of opening openings for depressurizing with the quasi-static nature of the explosion.

\section{Material and methods}

When interacting explosive load with construction structures, we will distinguish three modes of loading the structure depending on the ratio of the characteristic times of action of the load (explosion) and the time of development of deformation in the structure. The pulse loading mode is characterized by the ratio: $t_{\mathrm{ex}} / \mathrm{t}_{\mathrm{c}} \leq 1$, the quasi-static loading mode is characterized by the ratio $t_{e x} / t_{c} \geq 10$, where $t_{e x}$ is the characteristic time of action of the explosive load, $t_{c}$ is the characteristic time of deformation of the structure. During pulse loading, the final deformation is determined by the explosive load pulse, during quasi-static loading, the deformation is determined by the maximum explosion pressure value.

The intermediate values of $t_{e x} / t_{c}$ correspond to the dynamic loading mode, at which the final deformation is determined by the joint influence of the pulse and maximum pressure.

The limits given for the separation of modes are conditional, and the accuracy of the results is higher, the better the specified conditions are met.

Next, the case of a quasi-stationary explosion with the model load presented in Fig. 1.

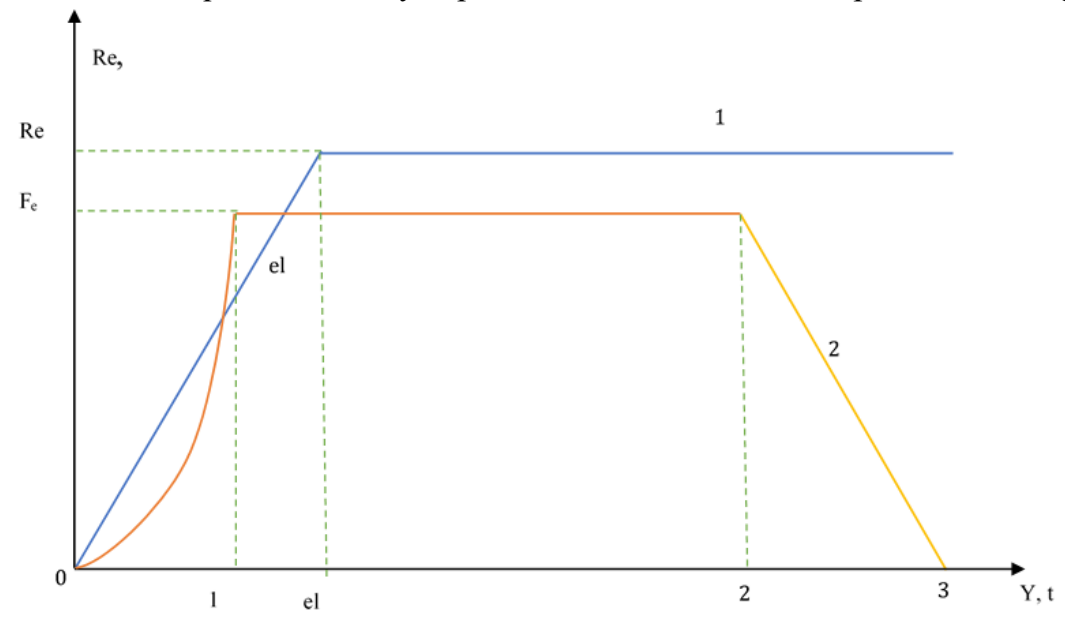

Fig. 1. Resistance diagram - deformation - 1. Model load at internal explosion - 2, Re equivalent resistance, $\mathrm{F}_{\mathrm{e}}$ - equivalent load. 
This load is constructed as follows: From the ratio:

$$
\Delta P_{2}=\frac{U_{g 1}^{2}(\sigma-1)^{2} K_{f}^{2} V_{0}^{2 / 3} \rho_{0}}{2 S_{0}^{2} K_{n}^{2} \sigma}
$$

Overpressure $\Delta \mathrm{P}_{2}$ in volume is determined if open area of discharge openings $\mathrm{S}_{0}$ is specified.

The second version is that the required area of open openings $\mathrm{S}_{0}$ is determined, if the permissible pressure $\Delta \mathrm{P}_{2}$ is set, such that the bending moment in the most dangerous section during static loading with such a pressure value does not exceed the internal plastic moment of resistance $M_{1}$.

Pressure $\Delta \mathrm{P}_{2}$ is implemented at the time of the maximum burning area which is equal to $K_{f} V_{0}{ }^{2 / 3}$ Here $\mathrm{V}_{0}$ is the free volume of the room, $\mathrm{K}_{\mathrm{f}}$ is the coefficient that takes into account the shape of the room in the formation of the maximum combustion area, $U_{\mathrm{g} 2}$ is the combustion speed at the moment of the maximum flame area, $K_{n}=0.6$ is the flow coefficient that takes into account the narrowing of the effluent gas jet compared to the area of the opening, $\sigma$ - the degree of expansion of the gases during combustion, $\rho_{0}-$ is the initial density of the combustible mixture. When maximum combustion area is reached, pressure peak is realized. Another peak of pressure $\Delta \mathrm{P}_{1}$ is realized during opening of safety structures, when the area for outflow has not yet reached $\mathrm{S}_{0}$.

The safety structure shall be considered effective if $\Delta \mathrm{P}_{1}=\Delta \mathrm{P}_{2}$.

How to ensure this condition when glass glazing is used as a safety structure is set out in [7]. In the case of opening of the inertial safety structure, this condition shall be met only [12]:

$$
\Delta \mathrm{P}_{2}>\Delta \mathrm{P}_{v}(1+\theta)^{3} k_{t}^{3}
$$

Where $\Delta \mathrm{P}_{\mathrm{v}}$ the pressure at which the bonds holding the safety structures are broken, the value $(1+\theta)$ is determined from the condition [12-13]:

$$
B=\frac{\frac{4}{\mathrm{~B}}=\frac{(1+\theta)^{5}}{5}-(1+\theta)+0,8}{\rho_{\Pi} X_{0} U g_{1} P_{0}^{2 / 3}\left[4 \pi \gamma(\sigma-1) \sigma^{2}\right]^{2 / 3}}
$$

Value $\mathrm{k}_{\mathrm{t}}$ - characterizes the time interval from the moment of opening of the opening to the moment of the first peak $\mathrm{P}_{1}$ and depends on parameter "B" according to Table 1 [12]:

Table 1. Dependence of parameter «B»

\begin{tabular}{|c|c|c|c|c|c|c|c|c|c|}
\hline $\mathrm{B}$ & 0.5 & 1 & 2 & 4 & 8 & 16 & 30 & $>30$ & $\infty$ \\
\hline $\mathrm{k}_{\mathrm{t}}^{3}$ & 1.025 & 1.03 & 1.035 & 1.075 & 1.085 & 1.12 & 1.07 & 1.2 & 1.8 \\
\hline $1+\theta$ & 2.16 & 1.91 & 1.71 & 1.54 & 1.41 & 1.31 & 1.26 & 1.2 & 1 \\
\hline
\end{tabular}

$\rho_{\mathrm{n}}$ - weight of unit area of the protective structure $\mathrm{kg} / \mathrm{m}^{2}, \mathrm{X}_{0}$ - depth of sealing of the protective structure in the opening, $\mathrm{U}_{\mathrm{g}}$ - burning speed at the moment of opening of the opening, $\mathrm{P}_{0}=10^{5} \mathrm{~Pa}$.

When constructing a model load, the maximum is taken as the most of the values $\Delta \mathrm{P}_{2}$ и $\Delta \mathrm{P}_{v}(1+\theta)^{3} k_{t}^{3}$, if the latter cannot be reduced. Pressure increase from 0 to maximum $\Delta \mathrm{P}_{\mathrm{m}}$ occurs according to the law:

$$
\Delta P(t)=\Delta \mathrm{P}_{m}\left(\frac{t}{t_{1}}\right)^{3}
$$

Where $t_{1}=t_{v}(1+\theta) k_{t}$ is the time of reaching $\Delta \mathrm{P}_{\mathrm{m}}$.

It should be noted that $t_{1}$ in (5) is underestimated compared to the real one, since when approaching the first peak there is a deviation from law (5).

After reaching the first peak, the pressure decreases, and then again rises to a value of $\Delta \mathrm{P}_{2}$. The nature of the decline from $\Delta \mathrm{P}_{1}$ and the rise to $\Delta \mathrm{P}_{2}$ are not sufficiently studied and therefore in the model load the maximum pressure value is reduced during time $t_{1}-t_{2}$ Fig. 1 .

The time interval $t_{12}=t_{1}-t_{2}$ can be estimated by analyzing the explosion. 
Obviously:

$$
t_{12}=\frac{r_{1}-r_{2}}{W_{2}}
$$

Where $r_{2}=\frac{K_{f} V_{0}{ }^{2 / 3}}{4 \pi}$ the effective radius of the flame front at the second peak, $r_{1}=$ $U_{\Gamma 1} \sigma t_{1}$ - the effective radius at the first peak, $w_{2}=U_{\Gamma 2} \sigma_{2}$ - the visible flame speed at section $t_{12}, \sigma_{2}$ - the effective degree of expansion, when determining it, the expiration of combustion products is taken into account, which reduces the visible flame speed. It depends on the explosion pressure and the ratio $S_{0} / V_{0}^{2 / 3}$. In case of explosions in laboratory conditions corresponding to real conditions $\sigma_{2}=0,6 \sigma$. The result is an estimate:

$$
\bar{t}_{12}=\bar{t}_{1} \frac{W_{1}}{W_{2}}\left[\frac{\left(P_{0} / \Delta P_{v}\right)^{1 / 3}}{k_{t}(1+\theta)}-1\right]
$$

As a result of the outflow of the combustion products, the effective rate and degree of expansion decreases, and the rate of combustion $\mathrm{U}_{\mathrm{g} 2}$ increases due to the occurrence of flame instability as a result of the action of the discharge wave, which is caused by the change of the flow of cold starting gases to hot combustion products. The effect of these processes is mutually compensated, and therefore $\frac{W_{1}}{W_{2}} \approx 1$ will be used for assessments.

The load decay time $t_{23}$ has little effect on the final deformation and the proposal for the duration of this section will be applied after consideration of the deformation in section $\mathrm{t}_{12}$.

To consider the deformation of the bent elements, a diagram of the bending resistance $\mathrm{R}_{\mathrm{y}}$ combined with the load $F_{m}(t)=\Delta P(t) b L$, is presented, Fig. 1

$\begin{array}{lll}R_{y}=k_{\ni} Y & Y<Y_{e l} & R_{m}=k_{\ni} Y_{e l}\end{array}$

$$
R_{y}=R_{m} \quad Y \geq Y_{e l}
$$

For hinged-supported beam:

$$
R_{m}=\frac{8 M_{p}}{L}, k_{\ni}=\frac{384 E J}{5 L^{5}}
$$

For a pinched beam at two ends:

$$
R_{m}=\frac{8\left(M_{p s}+M_{p m}\right)}{L}, k_{\ni}=\frac{307 E J}{L^{3}}
$$

For a beam fixed at one end and hinged-supported at the other end:

$$
R_{m}=\frac{4\left(M_{p s}+2 M_{p m}\right)}{L}, k_{\ni}=\frac{160 E J}{L^{3}}
$$

The following symbols are used in expressions (7) to (10):

$\mathrm{E}$ - modulus of elasticity, $\mathrm{J}$ - moment of inertia of section at bending, $\mathrm{Y}_{\mathrm{el}}-$ maximum deflection at the end of elastic stage, $\mathrm{M}_{\mathrm{p}}=Z_{p} \sigma_{T}$ - - maximum internal moment of bending resistance in section with plastic moment of bending resistance $Z_{\mathrm{p}}, \sigma_{\mathrm{T}}$ - dynamic yield strength of deformable material [15-16]. $\mathrm{M}_{\mathrm{ps}}$ is the plastic moment of resistance in the support section, $\mathrm{M}_{\mathrm{pm}}$ is the plastic moment of resistance in the middle section. For equivalent beam $\mathrm{M}_{\mathrm{p}}=\mathrm{M}_{\mathrm{pm}}=\mathrm{M}_{\mathrm{ps}}$ and therefore:

$$
R_{m}=\frac{16 M_{p}}{L} \quad R_{\mathrm{y}}=\frac{12 M_{p}}{L}
$$

When consideration of bending structures, it is convenient to use equivalent systems, that is, systems with reduced force and mass [17].

The equation of motion of the equivalent system in the elastic stage is described by the expression:

$$
\bar{Y}=\frac{Y}{Y_{e l}}, \bar{t}=\frac{t}{t_{c}} ; t_{c}=\sqrt{\frac{M_{l}}{k_{l}}} \quad \bar{Y}=B_{f}\left(\frac{\bar{t}}{\bar{t}_{1}}\right)^{3}
$$




$$
M_{l}=k_{n} M ; k_{l}=k_{L} k_{\ni} ; B_{f}=\frac{\Delta P_{m} b L}{R_{m}} ;
$$

The drive coefficients for mass and force for the case of the static deflection line in the elastic stage are:

$$
\begin{aligned}
& \left.\begin{array}{c}
k_{n}=0.5 \\
k_{L}=0.64
\end{array}\right\} \text { For hinged-supported beam } \\
& \left.\begin{array}{l}
k_{n}=0.41 \\
k_{L}=0.53
\end{array}\right\} \text { For a pinched beam at two ends } \\
& \left.\begin{array}{l}
k_{n}=0.45 \\
k_{L}=0.58
\end{array}\right\} \text { For a beam fixed at one end and hinged-supported at the other end/ } \\
& \text { In case of plastic deformation: }
\end{aligned}
$$$$
k_{n}=0.33, k_{L}=0.5 \text { for all types of beam anchoring. }
$$

This paper discusses deformation for the case $F_{m}<R_{m}$. For section $0-1$, where $\Delta P(t)=\Delta \mathrm{P}_{m}\left(\frac{t}{t_{1}}\right)^{3}$ at elastic deformation the solution (12) has the form [18].

$$
\bar{Y}=B_{f} \Phi ; \dot{\bar{Y}}=B_{f} \dot{\Phi} ;
$$

Where $\Phi=\frac{6}{{\overline{t_{1}}}^{3}}\left(\sin \bar{t}_{1}-\bar{t}_{1}+\frac{\bar{t}_{1}^{3}}{6}\right) ; \dot{\Phi}=\frac{6}{{\overline{t_{1}}}^{3}}\left(\cos \bar{t}-1+\frac{\bar{t}^{2}}{2}\right)$

In the 1- el section, that is, from the time $t_{1}$ of the end of pressure growth to the time of the end of elastic deformation, of the solution, the equation of motion has the form:

$$
\begin{array}{r}
\Delta \dot{\bar{Y}}_{1}=B_{f} \dot{\Phi} \cos \Delta \bar{t}+B_{f}(1-\Phi) \sin \Delta \bar{t} \\
\Delta \bar{Y}_{1}=B_{f} \dot{\Phi} \sin \Delta \bar{t}+B_{f}(1-\Phi)(1-\cos \Delta \bar{t})
\end{array}
$$

$\Delta \bar{Y}_{1}$ - is a dimensionless design offset after $\mathrm{t}_{1}$, that is, $\Delta Y=Y-Y_{1}$.

$\Delta \bar{t}-$ the time deducted from the moment $\mathrm{t}_{1}, \Delta \bar{t}=\bar{t}-\bar{t}_{1}$.

At $\Delta \bar{Y}_{1}=1-\bar{Y}_{1}=1-B_{f} \Phi$, the elastic stage of deformation in timepoint of $\Delta \bar{t}_{e l}=$ $\bar{t}_{e l}-\bar{t}_{1}$. comes to an end with _el-t. This moment is defined from the second equation (16) at $\Delta \bar{Y}_{1}=1-B_{f} \Phi$ and is equal:

$$
\Delta \bar{t}_{e l}=2 \operatorname{arctg} \frac{\Delta \bar{Y}_{e l}-\Delta \dot{Y}_{1}}{\left(2 B_{f}-1-B_{f} \Phi\right)}
$$

Next, the case is considered when the maximum deformation is plastic and ends in the section $\bar{t}_{1}-\bar{t}_{2}$ Fig.1 By the moment of maximum plastic deflection $Y_{m}$ the total strain energy is $R_{e} Y_{m}-\frac{1}{2} R_{e} Y_{e l}$. Operation of external force $\mathrm{F}(\mathrm{t})$ in section $0-Y_{m}$ is composed of operation in section 0 - $\mathrm{t}_{1}$, where force changes according to law (5) and operation of constant force $\mathrm{F}$ in section $Y_{1}-Y_{m}$. Energy conservation is written for an equivalent system, since the law will be universal for all types of beam anchoring.

$$
\begin{aligned}
& R_{e} Y_{m}-\frac{1}{2} R_{e} Y_{e l}=\frac{1}{2} k_{e} Y_{1}{ }^{2}-\frac{1}{2} m_{e} \dot{Y}_{1}^{2}+F_{e}\left(Y_{m}-Y_{1}\right) \\
& R_{e}=k_{e} R_{m}, \quad m_{e}=k_{n} M, \quad F_{e}=k_{e} F
\end{aligned}
$$

The result for the ductility coefficient is:

$$
\mu=\frac{1+B_{f}{ }^{2}\left(\Phi_{1}{ }^{2}+{\dot{\Phi_{1}}}^{2}-2 \Phi_{1}\right)}{2\left(1-B_{f}\right)}
$$

This expression is fair, that is it can be used if the maximum deformation is implemented until recession of loading $t_{2}$.

It follows from the expression (17) that in the equivalent systems carried out equal ductility coefficients are obtained for the same values $B_{f}$ and $t_{1}^{-}$. However, it should be borne in mind that the resistance forces $\mathrm{R}_{\mathrm{m}}$ and $\mathrm{R}_{\mathrm{e}}$ and the ultimate elastic deformations are different for different cases of fixation of beams. As a result, absolute deflections of $Y_{m}=\mu_{\infty} Y_{e l}$ turn out different. 
If in the expression (16) of conservation of energy instead of $\mathrm{Y}_{\mathrm{m}}$ substitute $\mathrm{Y}_{\mathrm{el}}$, then you can get the value of the velocity at the moment of the end of elastic deformation $\bar{Y}_{e l}$, given that $\overline{Y_{e l}}=1$. The result is:

$$
{\dot{Y}_{e l}}^{2}=B_{f}{ }^{2}\left(\Phi^{2}+\dot{\Phi}^{2}-2 \Phi\right)+2 B_{f}-1
$$

The plastic deformation step is described by:

Stop time after the start of plastic deformation

$$
\dot{Y}_{n}=\bar{Y}_{e l}-\left(1-B_{f}\right) \Delta \bar{t}
$$

$$
\Delta \bar{t}_{n}=\frac{\dot{Y}_{e l}}{\left(1-B_{f}\right)}
$$

As a result, ductility factor:

$$
\mu_{\infty}=1+\frac{\dot{Y}_{e l}{ }^{2}}{2\left(1-B_{f}\right)}
$$

It is easy to make sure using (18) that the expressions (17) and (21) match. These expressions are obtained on the assumption that the braking time during plastic deformation is $\Delta \bar{t}_{n}$ less than the action time of the maximum explosive load at the stage of plastic deformation, that is,

$$
\Delta \bar{t}_{n}<\bar{t}_{12}-\left(\bar{t}_{e l}-\bar{t}_{1}\right)=\Delta \bar{t}_{p l}
$$

If this condition is not met, the braking time in the area where the external load is maximum is $\bar{t}_{1}-\bar{t}_{e l}$, and deformation speed at the time of $\bar{t}_{2}$ is equal:

$$
\overline{\dot{Y}}_{2}=\bar{Y}_{e l}-\left(1-B_{f}\right)\left(\bar{t}_{2}-\bar{t}_{e l}\right)
$$

By the time $t_{2}$. the strain will be:

$$
\bar{Y}_{23}=\bar{Y}_{2}=1+\left[\bar{Y}_{e l}-\frac{\left(1-B_{f}\right)}{2}\left(\bar{t}_{2}-\bar{t}_{e l}\right)\right]\left(\bar{t}_{2}-\bar{t}_{e l}\right)
$$

Finally, in the pressure drop section 2-3, the movement of the structure is determined by the following ratios:

where $\Delta \overline{t_{2}}$ - is counted from $\bar{t}_{2}$,

$$
{\overline{Y_{23}}}_{23} \overline{\dot{Y}}_{2}-\left(1-B_{f}\right) \Delta \bar{t}_{2}-\frac{B_{f} \Delta{\overline{t_{2}}}^{2}}{2 \bar{t}_{23}}
$$

$\bar{t}_{23}$ - pressure drop time from maximum value $\Delta \mathrm{P}_{\mathrm{m}}$ to 0 .

During internal explosions, the pressure drop process was not studied in detail. However, it can be argued that large values of $\bar{t}_{23}$ correspond to large deformations in this area. With small values of $\bar{t}_{23}$, deformation may not end in this area. But in general, the difference in deformation in this area weakly affects the total deformation. Here it is proposed to accept $\bar{t}_{23}=\frac{\overline{\dot{Y}_{2}}}{1-\frac{B_{f}}{2}}$. At this value $\bar{t}_{23}$, the structure stops just at the moment $\bar{t}_{3}$. At the same time, the displacement of the structure in this section $\Delta \bar{Y}_{23}$ corresponds to:

$$
\bar{Y}_{23}=\frac{{\overline{Y_{2}}}^{2}}{2\left(1-\frac{B f}{2}\right)}
$$

\section{Results}

It was previously stated that this work considers the conditions leading to plastic deformations, provided that the force factor $B_{f}=\frac{F}{R_{m}}<1$. And it means that at the end of an elastic stage of deformation the design still has kinetic energy, that is $\overline{\dot{Y}}_{e l}>0$.

Table 2 shows the results of calculations of the velocity of the studied structure at the end of the elastic deformation stage for various combinations of the force factor $\mathrm{B}_{\mathrm{f}}$ and the explosion pressure rise time. 
Table 2. Velocity of the studied structure at the end of the elastic deformation stage

\begin{tabular}{|c|c|c|c|c|c|c|c|c|c|c|}
\hline$B_{f} \backslash \bar{t}_{1}$ & 0.5 & 1 & 2 & 3 & 4 & 5 & 7 & 10 & 20 & 30 \\
\hline 0.5 & - & - & - & - & - & - & - & - & - & - \\
\hline 0.6 & 0.443 & 0.432 & 0.387 & 0.310 & 0.198 & - & - & - & - & - \\
\hline 0.7 & 0.629 & 0.618 & 0.576 & 0.510 & 0.425 & 0.327 & 0.065 & - & - & - \\
\hline 0.8 & 0.770 & 0.759 & 0.715 & 0.646 & 0.560 & 0.466 & 0.288 & 0.127 & - & - \\
\hline 0.9 & 0.890 & 0.878 & 0.829 & 0.754 & 0.662 & 0.562 & 0.382 & 0.247 & 0.091 & - \\
\hline 0.95 & 0.944 & 0.930 & 0.880 & 0.800 & 0.704 & 0.600 & 0.413 & 0.277 & 0.134 & 0.088 \\
\hline$B_{e l}$ & 0.501 & 0.505 & 0.519 & 0.542 & 0.573 & 0.612 & 0.695 & 0.772 & 0.869 & 0.909 \\
\hline
\end{tabular}

Determination of speed of $\dot{Y}_{e l}$ is executed by _el based on the ratio of (18).

Therefore, the ratio is calculated the limit value of the force factor $\mathrm{B}_{\mathrm{el}}$, at which the deformation does not go into plastic for the specified load build-up time. This value is located in the last line of Table 2.

Table 3 gives the parameters often used in the calculation to characterize deformation at the load build-up stage, depending on the dimensionless load build-up time.

Table 3. Characterize deformation at the load build-up stage

\begin{tabular}{|c|c|c|c|c|c|c|c|c|c|c|}
\hline $\bar{t}_{1}$ & 0.5 & 1 & 2 & 3 & 4 & 5 & 7 & 10 & 20 & 30 \\
\hline$\Phi$ & 0.0125 & 0.0488 & 0.182 & 0.365 & 0.054 & 0.714 & 0.889 & 0.937 & 0.986 & $\begin{array}{c}0.99 \\
3\end{array}$ \\
\hline$\dot{\Phi}$ & 0.124 & 0.242 & 0.458 & 0.558 & 0.595 & 0.566 & 0.424 & 0.289 & 0.200 & $\begin{array}{c}0.09 \\
98\end{array}$ \\
\hline $\begin{array}{l}\Phi^{2} \\
+\dot{\Phi}^{2}\end{array}$ & 0.0155 & 0.0609 & 0.242 & 0.444 & 0.660 & 0.829 & 0.970 & 0.961 & 0.994 & 0.99 \\
\hline $\begin{array}{l}\Phi^{2} \\
+\dot{\Phi}^{2} \\
-2 \Phi\end{array}$ & 0.0095 & 0.0368 & 0.140 & 0.286 & 0.447 & 0.598 & -0.808 & -0.912 & -0.977 & - \\
\hline
\end{tabular}

Table 4 shows the maximum possible values of ductility coefficients $\mu_{\infty}$, which are realized when condition (24) is met, with sufficiently large values of $\bar{t}_{12}$.

Table 4. Ductility coefficients $\mu_{\infty}$,

\begin{tabular}{|c|c|c|c|c|c|c|c|c|c|c|}
\hline$B_{f} \backslash \bar{t}_{1}$ & 0.5 & 1 & 2 & 3 & 4 & 5 & 7 & 10 & 20 & 30 \\
\hline 0.95 & 9.91 & 9.67 & 8.74 & 7.4 & 5.96 & 4.6 & 2.73 & 1.77 & 1.18 & 1.065 \\
\hline 0.9 & 4.96 & 4.85 & 4.44 & 3.84 & 3.18 & 2.58 & 1.74 & 1.3 & 1.04 & 0.99 \\
\hline 0.8 & 2.48 & 2.44 & 2.28 & 2.04 & 1.78 & 1.54 & 1.21 & 1.04 & 0.94 & 0.92 \\
\hline 0.7 & 1.66 & 1.64 & 1.55 & 1.45 & 1.3 & 1.18 & 1.01 & 0.92 & 0.87 & 0.86 \\
\hline 0.6 & 1.25 & 1.23 & 1.19 & 1.12 & 1.05 & 0.98 & 0.89 & 0.84 & 0.81 & 0.805 \\
\hline
\end{tabular}

To fill with contents assessment (22) and to use expression (23) it is necessary to determine the size $\Delta \bar{t}_{e l}=\bar{t}_{e l}-\bar{t}_{1}$.

This value is shown in Table 5 .

Table 5. Determine the size $\Delta \bar{t}_{e l}=\bar{t}_{e l}-\bar{t}_{1}$.

\begin{tabular}{|c|c|c|c|c|c|c|c|c|c|c|}
\hline$B_{f} \backslash \bar{t}_{1}$ & 0.5 & 1 & 2 & 3 & 4 & 5 & 7 & 10 & 20 & 30 \\
\hline 0.95 & 1.5 & 1.37 & 1.14 & 0.91 & 0.83 & 0.55 & 0.376 & 0.474 & 1.98 & 0.32 \\
\hline 0.9 & 1.56 & 1.44 & 1.16 & 0.98 & 0.795 & 0.65 & 0.61 & 0.59 & & \\
\hline 0.8 & 1.7 & 1.58 & 1.31 & 1.15 & 0.987 & 0.88 & 0.665 & & & \\
\hline 0.6 & 2.2 & 2.07 & 1.78 & 1.8 & 1.75 & & & & & \\
\hline 0.5 & \multicolumn{10}{|c|}{ Deformation only in elastic stage. } \\
\hline
\end{tabular}


The following is an example of determining the development of deformation during an internal explosion.

Example 1.

$B_{f}=0,95, \mathrm{~B}=4, \bar{t}_{1}=4, \dot{Y_{1 e l}}=0.704$ (Table 2) и $\Delta \bar{t}_{e l}=0,83$ (Table 5), $\mathrm{P}_{\mathrm{v}}=1,2 \mathrm{kPa}$, $\bar{t}_{12}=1,75 \mathrm{t}_{1}$.

Time of plastic deformation in section $\bar{t}_{12}$ :

$\overline{\Delta t}_{p l}=\bar{t}_{12}-\overline{\Delta t}_{e l}=1.75 * 4-0.83=6.17$

Deformation rate to moment $\bar{t}_{1}$ of (25)

$\overline{\dot{Y}}_{2}=\overline{\dot{Y}}_{e l}-\left(1-B_{f}\right)\left(\bar{t}_{2}-\bar{t}_{e l}\right)=0.3955$

Deformation at moment $\bar{t}_{2}$ of (24)

$\bar{Y}_{2}=1+\left(0.704-\frac{0.05 * 6.17}{2}\right) 6.17=4.4$

Further, taking into account (28), deformation in the pressure drop section 2-3 is determined.

$\Delta \bar{Y}_{23}=\frac{\bar{Y}_{2}}{2\left(1-{ }^{B_{f} / 2}\right)}=0.149$

Total deformation:

$\mu_{\Sigma}=4.44+0,149=4,59<\mu_{\infty}=5,96$

The total deformation in this case is less than the deformation, obtained with a larger value $\bar{t}_{12}$.

Example 2.

$B_{f}=0,9, \mathrm{~B}=4, \bar{t}_{1}=5, \dot{Y_{1 e l}}=0.562, \Delta \bar{t}_{e l}=0.65$

Full braking time:

$\overline{t_{n}}=\frac{0.562}{1-0.9}=5.62$

Plastic Strain Time: $t_{p l}=t_{12}-\Delta t_{e l}=8.1$

Since $\overline{t_{n}}<\overline{t_{p l}}$ that is, the design stops before $\overline{t_{2}}$, and therefore $\mu_{\Sigma}=\mu_{\infty}=2,58$ Table 5 .

Example 3.

$B_{f}=0,95, \mathrm{~B}=0.5, \bar{t}_{1}=0.5, \dot{Y_{e l}}=0.994, \Delta t_{e l}=1.5, \mathrm{P}_{\mathrm{v}}=1,2 \mathrm{kPa}$.

From (6) $\bar{t}_{12}=0.53$.

This time is less than $\bar{t}_{e l}$, so the deformation in this area remains elastic and at the end of time $\bar{t}_{12}=0,53$ according to (14) $\dot{\bar{Y}}=0.58, \bar{Y}_{2}=0.2$.

Thus, the period of load decline at the moment $\bar{t}_{2}$ deformation is still elastic.

There is no experimental data on pressure drop under such conditions, then the operation of the external load is determined by the average value of this load, and then from the conservation of energy it is obtained:

$R_{l} Y_{m}-\frac{R_{l} Y_{e l}}{2}=\frac{1}{2} k_{e} Y_{2}^{2}+\frac{1}{2} m_{e} \dot{Y}^{2}+\frac{F_{e}}{2}\left(Y_{m}-Y_{2}\right)$ or

$\mu_{\Sigma}\left(1-\frac{3 B_{f}}{4}\right)=\frac{1}{2}\left(\bar{Y}_{2}^{2}+{\dot{Y}_{2}^{2}}^{2}\right)-\frac{3 B_{f}}{4} \bar{Y}_{2}, \mu_{\Sigma}=1.8$

In the energy conservation record, assuming the possibility of plastic deformations at the pressure drop stage. The result is very specific with the result $\mu_{\infty}=9,91$ for $\bar{t}_{1}=0,5$, and $B_{f}=0.95$.

The paper presents possible methods for calculating the load capacity of beam structures during an internal explosion.

When analyzing the deformation of beam structures during quasi-static explosions, a model load with a rise phase corresponding to the first peak was used, and the maximum load was taken by most of the two peaks. Such a load is conservative, since the maximum pressure 
value for the constant pressure phase is selected, which in turn is also a conservative estimate of the dynamics of pressure between peaks.

Both time of increase of pressure of $\bar{t}_{1}$ and time between $\mathrm{t}$ pressure peaks $\bar{t}_{12}$. has considerable impact on the nature of loading. At high pressure times, the loading pattern is quasi-static, the ductility coefficient exceeds the value $\mu_{\infty}>2$ only for the power factor $B_{f}>0.9$.

With a force factor, $B_{f}>0.5$ deformation does not change to plastic never. The $\mu_{\infty}$ strain level increases monotonically as the force factor increases, as shown in Table 4. However, the time interval $\bar{t}_{12}-\overline{\Delta t}_{e l}$ is often insufficient to complete plastic deformation under maximum model load conditions. The time interval $\bar{t}_{12}$ decreases with a decrease in the volume of the room and an increase in the rate of explosive combustion due to a decrease in $\bar{t}_{1}$ (see (6). The second multiplier in (6) also decreases with an increase in the combustion rate and a decrease in volume. This follows from (3-4). However, $\overline{\Delta t}_{e l}$ grows with a decrease in $\bar{t}_{1}$ Therefore, even at high $\mathrm{B}_{\mathrm{f}}$ values (Example 3 ), deformation may not proceed to the plastic stage.

However, it must be kept in mind that in the conditions of the small size of parameter B it is necessary to pay less than unit for preservation of a power factor of $B_{f}$.

\section{Conclusion}

In conclusion, it should be noted that the present work is essentially the first to address the deformation of the supporting structure in internal emergency explosions, taking into account the effect of safety structures. This action takes into account both the design maximum pressure and the time interval between pressure peaks. The paper considers the case when the explosive load controlled by the action of protective structures meets the requirement of $B_{f}<1$, which in turn means the impossibility of plastic deformation at the stage of pressure growth at the beginning of the explosion. The model load allows you to make a conservative estimate of deformation, since the maximum pressure acts throughout the time between peaks, and it corresponds to the pressure at a larger peak. Design conditions are not taken into account in the work, reliability issues are not considered.

They are left to the discretion of the persons concerned.

The author believes that further experimental studies of internal explosions using safety structures will help to take into account the nature of the pressure change between peaks and clarify the calculation of deformation in the pressure drop section.

\section{References}

1. Set of rules 12.13130.2009. Determination of categories of rooms, buildings and external installations on explosion and fire hazard. Moscow, All-Russian Research Institute for Fire Protection of Emercom of Russia Publ., 2009. 28 p. (in Russian).

2. Set of rules 4.13130.2013. Systems of fire protection. Restriction of fire spread at object of defense. Requirements to special layout and structural decisions. Moscow, AllRussian Research Institute for Fire Protection of Emercom of Russia Publ., 2013. 184 p. (in Russian).

3. Set of rules 56.13330.2011. Production buildings. Moscow, Ministry of Regional Development of Russia Publ., 2011. 18 p. (in Russian).

4. Set of rules 20.13330.2010. Loads and impacts. Ministry of Regional Development of Russia Publ., 2010. 80 p. (in Russian).

5. Technique of determination of settlement sizes of fire risk on production objects. Approved of order of Emercom of Russia on July 10, 2009 No. 404 
6. V. A. Gorev, A. I. Plotnikov. Stability at external emergency explosions. Urban building complex and safety of life support of citizens. Proceedings of scientific and practical conference. Moscow, ASV Publ., (2005), pp. 32-45 (in Russian).

7. N. N. Popov, B. S. Rastorguev, A. V. Zabegaev. Calculation of designs on inertial express reaction. Moscow, Vysshaya shkola Publ., (1992). 319 p. (in Russian)

8. B. S. Rastorguev, A. I. Plotnikov, D. Z. Khusnutdinov. Design of buildings and structures under emergency explosive impacts, Moscow, (2007).152 p.

9. L. P. Pilyugin. Designs of constructions of explosive productions (theoretical bases of design). Moscow, Stroyizdat Publ., (1988). 316 p. (in Russian).

10. L. P. Pilyugin. Maintenance explosion proof buildings with relief designs. Moscow, Pozhnauka Publ., (2000). 224 p. (in Russian).

11. U. Baiker, P. Koks. Explosive phenomena. Assessment and consequences. - Moscow. (1985).

12. V. A. Gorev, V. V. Molkov. On the dependence of internal explosion parameters on the installation of safety structures in the apertures of the protecting walls of industrial and residential buildings. Pozharovzryvobezopasnost Fire and Explosion Safety, 2018, vol. 27, no. 10 , pp. 6-25.

13. V.A. Gorev. Ensuring explosion safety of residential buildings. International Scientific Conference Environmental Science for Construction Industry - ESCI (2018). V. 193, 2018

14. V. A. Gorev, V. V. Belyaev, V. N. Fedotov. Condition of the beginning of vibrational gas combustion in an unsealed vessel of rectangular shape. Combustion, Explosion, and Shock Waves, (1989), vol. 25, no. 1, pp. 31-34.

15. P. Simmonds. the Dynamics of inelastic structures. Mechanics. No. 29. M. Mir. 1982g. 224 р. Аварии и катастрофы.

16. K. E. Kochetkov, V. A. Kotlyarovsky. Accidents and catastrophes.Prevention and elimination of consequences. DIA, (1995).

17. J. M. Biggs. Introduction to Structural Dynamics / J. M. Biggs // McGraw-Hill, New York, N.Y. - (1964).

18. V.A. Gorev. Deformation of beam structures in an internal explosion. Journal of Physics: Conference Series, (2020), 1425(1). 